\title{
Efectos de la covid-19 en la educación superior en línea en el estado de Guerrero, México: percepción de los estudiantes
}

The COVID-19 Effects on Online Higher Education at The State of Guerrero, Mexico: The students Perception

Efeitos da covid-19 no ensino superior online no estado de Guerrero, México: percepção dos alunos

\footnotetext{
Delia Inés Domínguez García

Universidad Autónoma de Guerrero, México deliadomgar@yahoo.com.mx https://orcid.org/0000-0002-7275-7120

Fernando Torres Romero Centro Universitario Villavicencio, México Villavic.pos@yahoo.com.mx https://orcid.org/0000-0002-4389-3369

Rodrigo Rosario Cruz Universidad Autónoma de Guerrero, México rockdrig@yahoo.com.mx https://orcid.org/0000-0002-4695-7129
} 


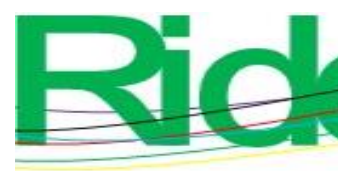

Revista Iberoamericana para la Investigación y el Desarrollo Educativo ISSN 2007- 7467

\section{Resumen}

El avance de la covid-19, ocasionada por el virus Sars-CoV-2, obligó a los países de América Latina y el Caribe a cerrar la puerta de las instituciones educativas en los niveles de preescolar, primaria y secundaria. En México, se cerraron también las universidades públicas y privadas; por esta razón, es importante hacer un diagnóstico del impacto de las medidas de confinamiento y distanciamiento social. El objetivo de este estudio fue analizar los efectos de la covid-19 en el desempeño académico y en la salud emocional de la población estudiantil de la Escuela de Ciencias Naturales de la Universidad Autónoma de Guerrero, en el contexto de la transición de la educación presencial a un modelo de educación en línea.

Los resultados del estudio muestran que $53.4 \%$ de los encuestados disponen de un espacio cómodo para atender sus clases en línea, pero solo $25 \%$ cuenta con un ambiente suficientemente agradable para lograr la concentración necesaria para un aprendizaje efectivo, $52.3 \%$ cuenta con los materiales necesarios para la realización de sus actividades, $26.1 \%$ tiene contacto con el profesor y $56.8 \%$ solo tiene contacto con algún compañero de grupo para resolver las dudas del curso, Asimismo, $43.2 \%$ tiene un dispositivo digital con mala conectividad, $27.3 \%$ con una buena conexión, y $3.4 \%$ no tiene computadora ni conexión a internet; igualmente, $23.9 \%$ carece de un espacio adecuado para realizar sus actividades, $15.9 \%$ no está capacitado en materia de competencias digitales y $14.8 \%$ no tiene computadora, lo que refleja un escenario poco promisorio, puesto que $28.4 \%$ de los encuestados tienen poco contacto con el profesor y $10.2 \%$ casi nunca. De acuerdo con estos resultados, se concluye que los factores que ponen en riesgo la continuidad de la educación son la carencia de una computadora, el acceso limitado a internet, la limitada interactividad por parte de los profesores con los alumnos y el desempeño de actividades extracurriculares prioritarias para apoyar la economía familiar, lo cual pone de relieve la brecha tecnológica entre las diferentes regiones geográficas.

Palabras clave: coronavirus, covid-19, educación en línea, educación superior, pandemia. 


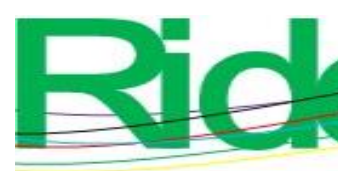

Revista Iberoamericana para la
Investigación y el Desarrollo Educativo ISSN 2007 - 7467

\section{Abstract}

The advance of COVID-19 caused by the SARS-CoV-2 virus, has forced the Latin America and the Caribbean countries to close the door of educational institutions at the preschool, elementary and Junior High School levels. In Mexico, public and private universities were also closed. For this reason, it is important to carry out a diagnosis of the impact of confinement and social distancing measures. The aim of this study was to analyze the effects of COVID-19 on the academic performance and emotional health of the student population of the School of Natural Sciences (ESCN) from the Autonomous Guerrero State University, within the context of the transition from face-to-face education to an online educational model.

The study was conducted on a sample of 88 students by applying an online survey. The results of the study show that $53.4 \%$ of the respondents have a comfortable space to attend their online classes, but only $25 \%$ have a sufficiently pleasant environment to achieve the concentration necessary for effective learning, $52.3 \%$ have the materials necessary to carry out their activities, $26.1 \%$ have contact with the teacher and $56.8 \%$ only have contact with a classmate to answer questions about the course, $43.2 \%$ have a digital device with poor connectivity, $27.3 \%$ have a good connectivity, and $3.4 \%$ do not have a computer nor internet access, $23.9 \%$ have no adequate space to carry out their activities, $15.9 \%$ has not enough training on digital skills and $14.8 \%$ do not have a computer, which reflects an unpromising scenario since, $28.4 \%$ of all those surveyed have little contact with the teacher almost always and $10.2 \%$ almost never. According to these results, it is concluded that the factors that put the continuity of education at risk are: The lack of a computer, limited access to the Internet, limited interactivity by teachers with students and the performance of extracurricular activities to support the family economy, which highlights the technological gap between the different geographic regions.

Keywords: coronavirus, covid-19, online education, higher education, pandemic. 


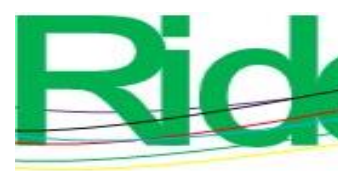

Revista Iberoamericana para la
Investigación y el Desarrollo Educativo ISSN 2007- 7467

\section{Resumo}

O avanço da covid-19, causado pelo vírus Sars-CoV-2, obrigou os países da América Latina e do Caribe a fechar as portas das instituições de ensino nos níveis pré-escolar, primário e secundário. No México, as universidades públicas e privadas também foram fechadas; Por esse motivo, é importante diagnosticar o impacto das medidas de confinamento e distanciamento social. O objetivo deste estudo foi analisar os efeitos da covid-19 no desempenho acadêmico e na saúde emocional da população estudantil da Faculdade de Ciências Naturais da Universidade Autônoma de Guerrero, no contexto da transição do presencial educação. a um modelo de educação online.

Os resultados do estudo mostram que 53,4\% dos entrevistados possuem um espaço confortável para assistir suas aulas online, mas apenas $25 \%$ possuem um ambiente agradável o suficiente para atingir a concentração necessária para um aprendizado eficaz, $52,3 \%$ possuem os materiais necessários para carregar fora de suas atividades, $26,1 \%$ têm contato com o professor e $56,8 \%$ só têm contato com um colega para tirar dúvidas sobre o curso. Da mesma forma, 43,2\% possuem dispositivo digital com conectividade ruim, 27,3\% com boa conexão. não possuir computador ou conexão com a internet; Da mesma forma, 23,9\% carecem de espaço adequado para realizar suas atividades, 15,9\% não são formados em habilidades digitais e 14,8\% não possuem computador, o que reflete um cenário pouco promissor, pois $28,4 \%$ dos entrevistados têm pouco contato com o professor e $10,2 \%$ quase nunca. De acordo com esses resultados, conclui-se que os fatores que colocam em risco a continuidade do ensino são a falta de computador, acesso limitado à Internet, interatividade limitada dos professores com os alunos e a realização de atividades extracurriculares. economia familiar, o que evidencia o fosso tecnológico entre as diferentes regiões geográficas.

Palavras-chave: coronavírus, covid-19, educação online, ensino superior, pandemia.

Fecha Recepción: Mayo 2021

Fecha Aceptación: Febrero 2022 


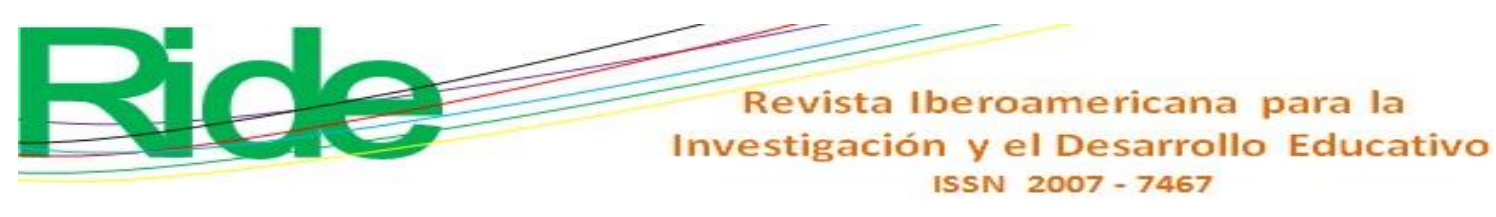

\section{Introducción}

Debido a la crisis mundial ocasionada por la pandemia de la covid-19, originada por el virus Sars-CoV-2, de manera súbita las escuelas y universidades de todo el mundo cerraron sus puertas, lo que afectó a 1570 millones de estudiantes en 191 países (Unesco, 2020a). Los cierres, como medida para contener la pandemia, llevaron a un despliegue de alternativas de solución para asegurar la continuidad educativa mediante la implementación de la educación en línea. Sin embargo, los obstáculos han sido múltiples, como la falta de conectividad, la incompatibilidad de los planes de estudio nacionales y la escasa formación del profesorado para afrontar esta "nueva normalidad" (Unesco, 2020b).

Por su parte, los estudiantes, que se encuentran en casa, deben compartir los dispositivos digitales y la red de internet que usa toda la familia, y tienen la necesidad de continuar sus actividades de aprendizaje de las diversas asignaturas inscritas a través de tareas, conferencias virtuales y una serie de deberes que se superponen. Además, quienes se enferman tienen que enfrentar los efectos de la pandemia en su propia salud, las emociones, actividades físicas y las propias de la juventud (The Chronicle of Higher Education, 2020).

Desde el 23 de marzo de 2020, el gobierno de México, a través de la Secretaría de Educación Pública, como una medida de protección para estudiantes, docentes y la población en general, determinó suspender las clases presenciales (Comisión Nacional para la Mejora Continua de la Educación [Conamece], 2020). Esto obligó a los profesores a emplear diversos instrumentos para seguir promoviendo el aprendizaje, por lo que tuvieron que cambiar su papel tradicional de enseñanza en el aula para convertirse en entes mediadores entre el alumno y la información facilitada mediante el uso de la tecnología (Quintero, 19 de mayo de 2020).

Ante el panorama de la covid-19, sin duda, se transformó la forma en la que se imparte la educación actualmente, ya que la escuela y el hogar se convirtieron en un mismo espacio (Anuies, 2020). Naturalmente, este cambio tan abrupto ha representado un enorme desafío para la equidad educativa con consecuencias que afectan la vida de los estudiantes más vulnerables (Villafuerte, 19 de marzo de 2020).

A más de un año de iniciada la pandemia, declarada por la Organización Mundial de la Salud (Cucinotta y Vanelli 2020), es necesario conocer los impactos que ha tenido el aislamiento social preventivo en los alumnos, así como los principales desafíos que se presentaron en el proceso de transformación de un modelo presencial a uno virtual (Ballén 


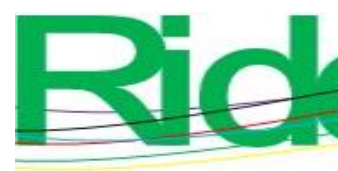

Revista Iberoamericana para la Investigación y el Desarrollo Educativo ISSN $2007-7467$

Cifuentes et al., 2021). Si bien los estudiantes son nativos digitales, no sucede lo mismo con los docentes, quienes en muchos casos aún tenían sus reservas frente al empleo de la tecnología en las aulas, aunque también cabe señalar que para otros esta ha sido la oportunidad para experimentar fórmulas creativas y resilientes con resultados asombrosos (Marín, 8 de junio de 2020).

La crisis mundial debido a la pandemia de covid-19 ha desencadenado un replanteamiento de la educación en todos los niveles, así como del uso intensivo de plataformas y recursos tecnológicos (Valverde-Berrocoso et al., 2021) ya sea de forma sincrónica o asíncrona (Moorhouse y Wong, 2021). Sin embargo, la premura del cambio en la metodología de la enseñanza, en diversas partes del mundo, ha visto esta transición como el experimento más audaz en materia de tecnología educativa por la falta de planeación y lo inesperado del evento (Unesco, 2020a). En consecuencia, es de suma importancia evaluar los resultados de las lecciones aprendidas para reforzar la inclusión, la innovación y la cooperación en la enseñanza (Giannini, 2020).

En este contexto, resulta importante tener en cuenta que el aprendizaje en las circunstancias que impone la pandemia presenta desafíos debido al cambio abrupto que implica la suspensión de las clases presenciales y la adaptación a la modalidad de educación en línea, es decir, “aprender desde casa” (Delgado, 6 de abril de 2020). A esto se suma que la didáctica universitaria en entornos virtuales de enseñanza es inapropiada (Santos-Baranda y Armas-Velasco, 2 de marzo de 2020; Quintero, 2020; Unesco, 2020c).

El paso repentino a la enseñanza en línea se implementó como una medida para garantizar la continuidad académica; sin embargo, este hecho ha implicado una serie de desafíos para las universidades. Fue necesario hacer cambios precipitados y, además, por un periodo incierto que aún no ha finalizado. Las decisiones que se toman en estas circunstancias corren el riesgo de no ser las mejores, por lo que resulta imperativo analizar la situación en la que nos encontramos ahora con el fin de evaluar y predecir las repercusiones futuras (Philip y Hans, 2020).

Con base en lo anterior, se plantea como objetivo analizar los efectos de la pandemia y el cierre de las instituciones educativas sobre el desempeño académico y la salud emocional de la población universitaria en el estado de Guerrero, México, en el contexto de la transición de la educación presencial a un modelo de educación en línea. 


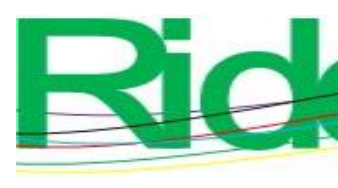

Revista Iberoamericana para la Investigación y el Desarrollo Educativo ISSN $2007-7467$

\section{Materiales y métodos}

El presente es un estudio exploratorio y descriptivo no experimental, realizado a través de la aplicación de un instrumento tipo encuesta en línea, dirigido a estudiantes de la Escuela Superior de Ciencias Naturales (ESCN) de la Universidad Autónoma de Guerrero (UAGro), en la transición de un sistema de educación presencial a un sistema en línea. Este fue diseñado para explorar la percepción sobre la efectividad en la instrumentación de las estrategias de enseñanza empleadas durante la emergencia sanitaria de la covid-19 y sus efectos psicosociales o alteraciones emocionales de la personalidad de los estudiantes; asimismo, se procuró precisar las modificaciones de la conducta provocadas por la pandemia y el estrés asociado al cambio de las estrategias para garantizar la continuidad de la educación.

El instrumento se hizo llegar a destinatarios inscritos en los grupos previamente seleccionados para el estudio vía correo electrónico, y se invitó a responderlo de forma voluntaria en la plataforma de Google Forms. El instrumento se envió a 95 estudiantes oficialmente inscritos en el octavo semestre de los programas educativos de Químico Farmacéutico Biólogo y Biología Experimental de la ESCN, de los cuales se devolvieron solo 88 cuestionarios $(n=88)$ a través del sistema en línea del Google Drive oficial utilizado por el sistema virtual de la UAGro, lo que representa $92.6 \%$ de respuestas recibidas.

El cuestionario se contestó en línea de manera voluntaria, por lo que se consideró que es una muestra no probabilística, intencional y por cuotas de estudiantes. Los resultados de Google Forms se exportaron a Google Sheets para generar las estadísticas y gráficas de los resultados.

Las opciones de respuesta de las preguntas del instrumento se enfocaron en cuatro dimensiones: 1) los hábitos y la percepción sobre el fenómeno de la covid-19, 2) las condiciones tecnológicas de los estudiantes, 3 ) los efectos emocionales del confinamiento y 4) las propuestas de innovaciones educativas para la mejora docente. La pregunta abierta se hizo al través de un análisis de las respuestas, lo que implicó leerlas, diferenciar los temas, clasificarlos y agruparlos semánticamente (van Dijk, 2000), para posteriormente agruparlos en categorías (Han, 2008) que permitieran identificar otras problemáticas a las que se están enfrentando los estudiantes, así como las propuestas concretas que tienen para que la universidad les brinde el apoyo pertinente. 


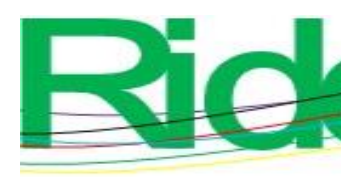

Revista Iberoamericana para la
Investigación y el Desarrollo Educativo
ISSN $2007-7467$

\section{Resultados}

Los resultados de este estudio fueron derivados de una encuesta enviada a través de la plataforma virtual Google Drive de la UAGro a 95 estudiantes del octavo semestre de los programas educativos de Químico Farmacéutico Biólogo y Biología Experimental de la ESCN, de los cuales se contestaron solo 88 cuestionarios $(n=88)$, lo que corresponde a $92.6 \%$ del total de formularios enviados.

Las estadísticas de los resultados de este estudio muestran que $56.8 \%$ de los estudiantes que participaron en la encuesta solo tienen contacto con algún compañero de grupo para resolver las dudas del curso, $53.4 \%$ disponen de un espacio cómodo para atender sus clases en línea, $52.3 \%$ cuentan con los materiales básicos necesarios para la realización de sus tareas académicas, $26.1 \%$ pueden establecer contacto con el profesor para solucionar sus dudas y $25 \%$ tienen en su hogar un ambiente de concentración óptimo para el desempeño y desarrollo de sus actividades educativas (figura 1).

Figura 1. Condiciones materiales y de comunicación con los que disponen en el hogar los estudiantes de la ESCN

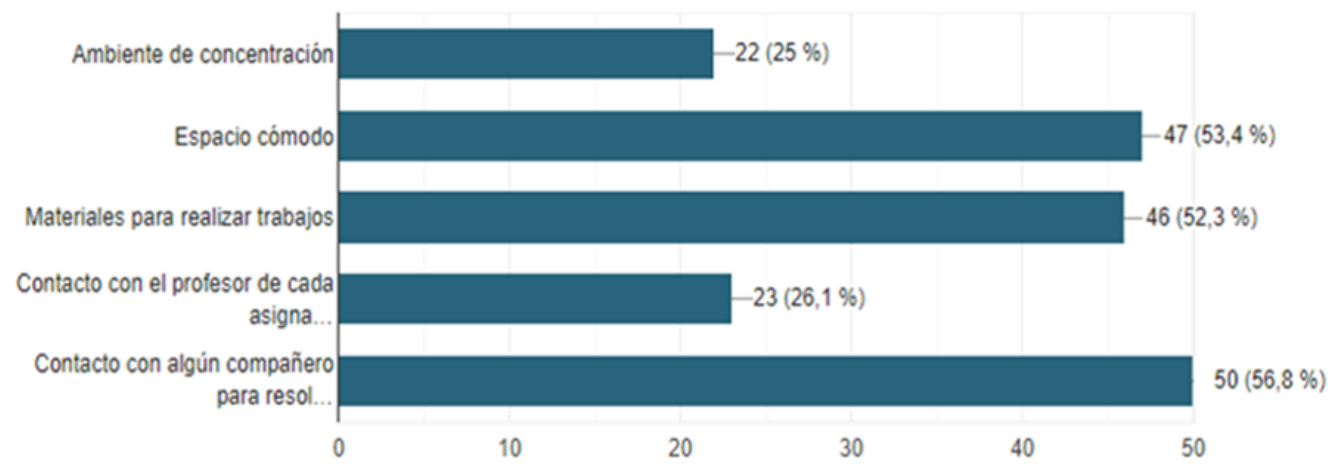

Fuente: Elaboración propia

Referente a la disponibilidad de dispositivos digitales y conectividad a una línea de red, $43.2 \%$ disponen de una computadora de pantalla mediana o tableta, pero tienen una conectividad irregular o mala, $27.3 \%$ tienen al menos una computadora o tableta y buena conectividad en su hogar, $26.1 \%$ cuenta solo con celular con conexión y $3.4 \%$ no tiene un dispositivo digital ni conexión a la red (figura 2). 

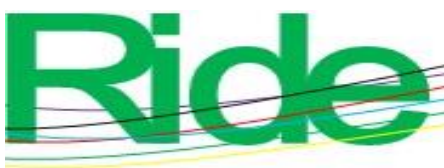

Revista Iberoamericana para la Investigación y el Desarrollo Educativo

ISSN 2007 - 7467

Figura 4. Actividades de acompañamiento, apoyo y retroalimentación a los estudiantes por parte de la planta docente de la escuela

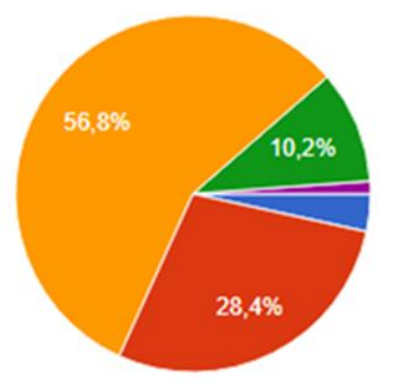

Fuente: Elaboración propia

En cuanto al nivel de preparación de los docentes con respecto a las herramientas, sitios y rutas para implementar las estrategias digitales de la educación en línea, fue otro de los rubros que se sometieron al escrutinio de los estudiantes y se encontraron los siguientes datos estadísticos: $46.6 \%$ percibe que la mayoría de los profesores están preparados, $35.2 \%$ que solo algunos lo están, $13.6 \%$ que pocos están preparados, $4.5 \%$ que todos lo están (figura 5).

Figura 5. Preparación de los docentes para implementar las estrategias digitales de la educación en línea

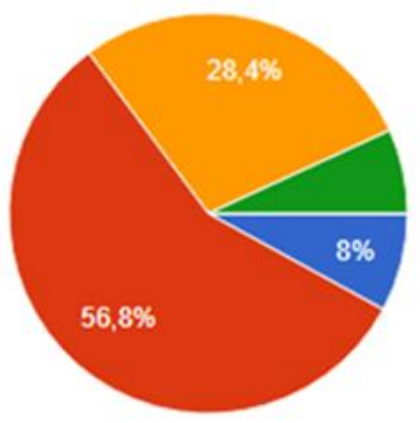

Excelentemente preparados

Moderadamente preparados

Significativamente preparados

Escasamente preparados

No estaban preparados

Fuente: Elaboración propia

En lo que se refiere a la planeación docente — asociada con la elaboración de los contenidos, tareas, dinámicas y evaluaciones - $50 \%$ perciben que la mayoría de sus profesores mostraron una apropiada planificación de clases, $31.8 \%$ indicó que solo 

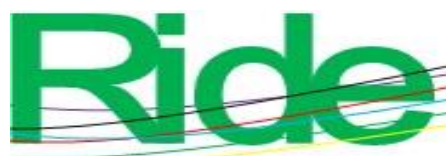

Revista Iberoamericana para la Investigación y el Desarrollo Educativo

ISSN 2007 - 7467

algunos, $9.1 \%$ que pocos y $9.1 \%$ que ninguno (figura 6).

Figura 6. Proporción de los integrantes de la comunidad docente que mostraron una planeación apropiada en los contenidos de las tareas, dinámicas y evaluaciones en el nuevo modelo de educación en línea
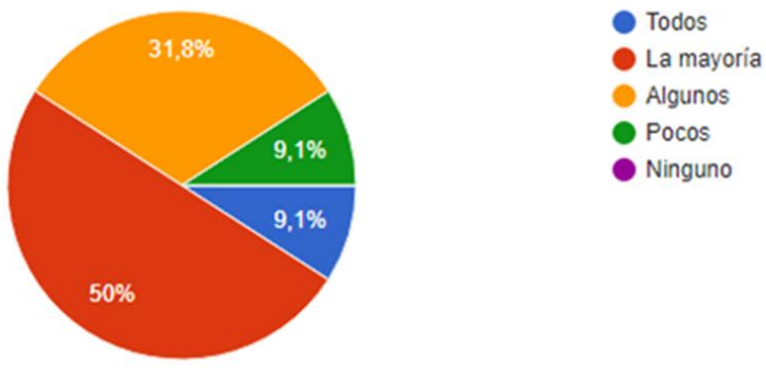

Fuente: Elaboración propia

En la nueva modalidad de educación en línea, $45.5 \%$ refieren que los profesores no proporcionaron las instrucciones durante las clases teóricas, prácticas y ejercicios asignados, $44.3 \%$ que la cantidad de tareas y actividades solicitadas por los profesores fue apropiada, $43.2 \%$ que el tiempo de entrega de las actividades académicas no fue suficiente, $36.4 \%$ percibió que la cantidad de tareas y actividades fue excesiva, $29.5 \%$ refirió que el tiempo para entregar las tareas fue suficiente, $20.5 \%$ indicó que los profesores proporcionaron explicaciones apropiadas para llevar a cabo los ejercicios teóricos y prácticos de los trabajos escolares, $6.8 \%$ que las tareas y actividades fueron muy difíciles y solo $4.5 \%$ mencionó que las tareas y actividades fueron muy fáciles (figura 7). 

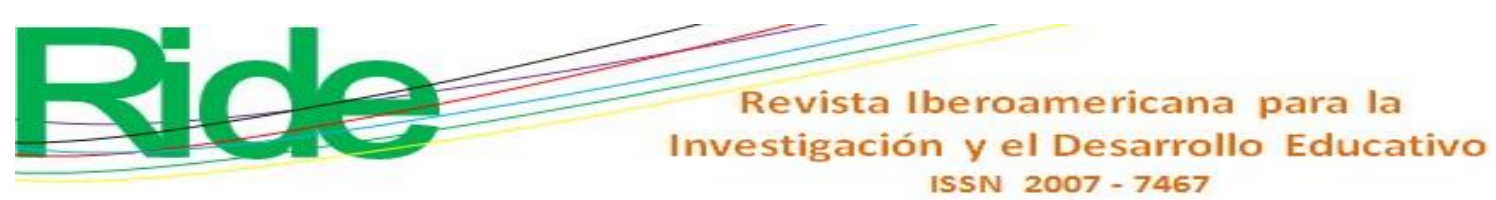

Figura 7. Principales motivos que dificultaron el proceso de enseñanza-aprendizaje asociados con el desempeño de las actividades docentes

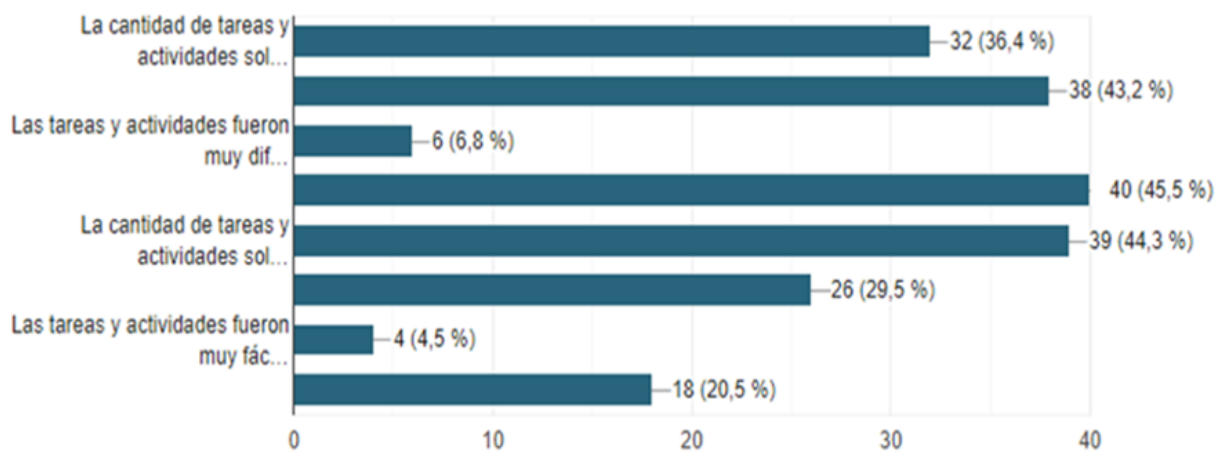

Fuente: Elaboración propia

Con respecto de los cambios de comportamiento y hábitos de vida de los estudiantes encuestados y la estabilidad emocional ocasionada por la covid-19, las estadísticas indican que $53.4 \%$ refieren que han cambiado mucho sus hábitos de vida, $45.5 \%$ que han cambiado poco y solo $1.1 \%$ no los ha modificado (figura 8 ).

Figura 8. Cambios de comportamiento y hábitos de vida de los estudiantes universitarios debido a la covid-19.

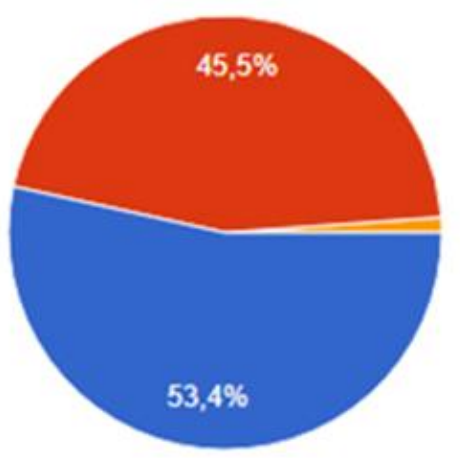

Sí, los he cambiado mucho

Si, aunque los he cambiado poco

No, no los he cambiado

Fuente: Elaboración propia

En este contexto, $45.5 \%$ de los encuestados considera que el brote de la covid-19 es extremadamente grave, $33 \%$ que es una enfermedad muy grave, $20.5 \%$ que es grave y solo $1.1 \%$ que es moderadamente grave (figura 9). 


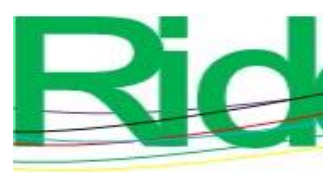

Revista Iberoamericana para la Investigación y el Desarrollo Educativo ISSN $2007-7467$

Figura 9. La covid-19 como una amenaza para la salud pública

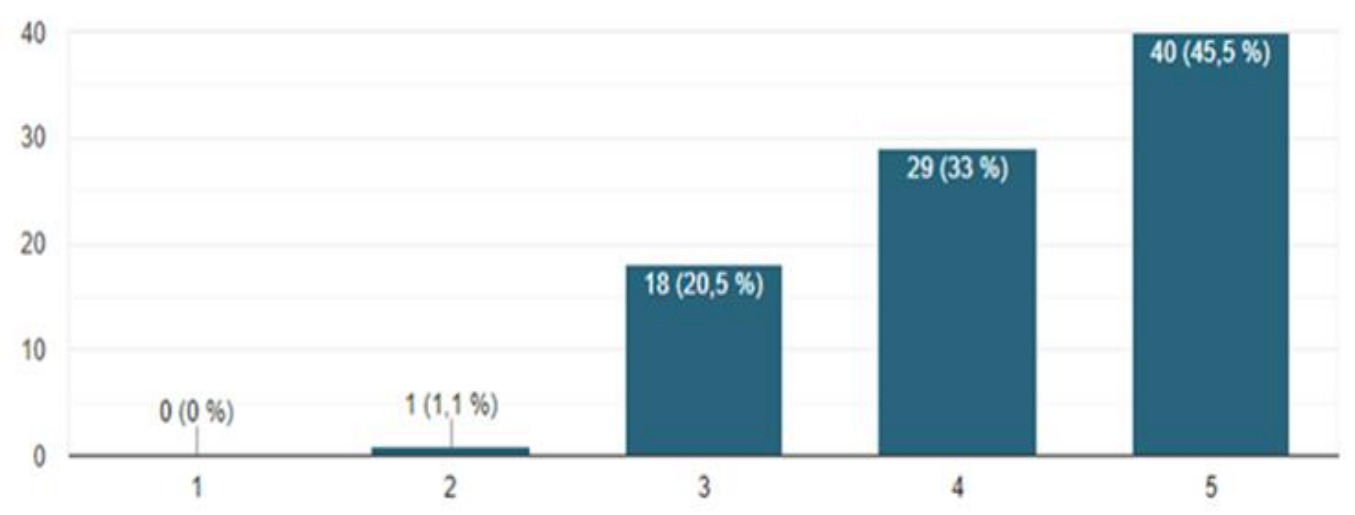

Fuente: Elaboración propia

Por último, el efecto emocional y de conducta ante la crisis generada por la pandemia refleja que $56.8 \%$ de los encuestados se sienten ansiosos y estresados, $38.6 \%$ asustados y preocupados, $30.7 \%$ felices de estar en casa, $29.5 \%$ molestos y frustrados, $23.9 \%$ aburridos, $15.9 \%$ solos, tristes y deprimidos, $14.8 \%$ acompañados, $12.5 \%$ tranquilos, $12.5 \%$ cansados y $4.5 \%$ entretenidos (figura 10).

Figura 10. Estado emocional de los estudiantes asociado a las medidas del confinamiento por la covid-19

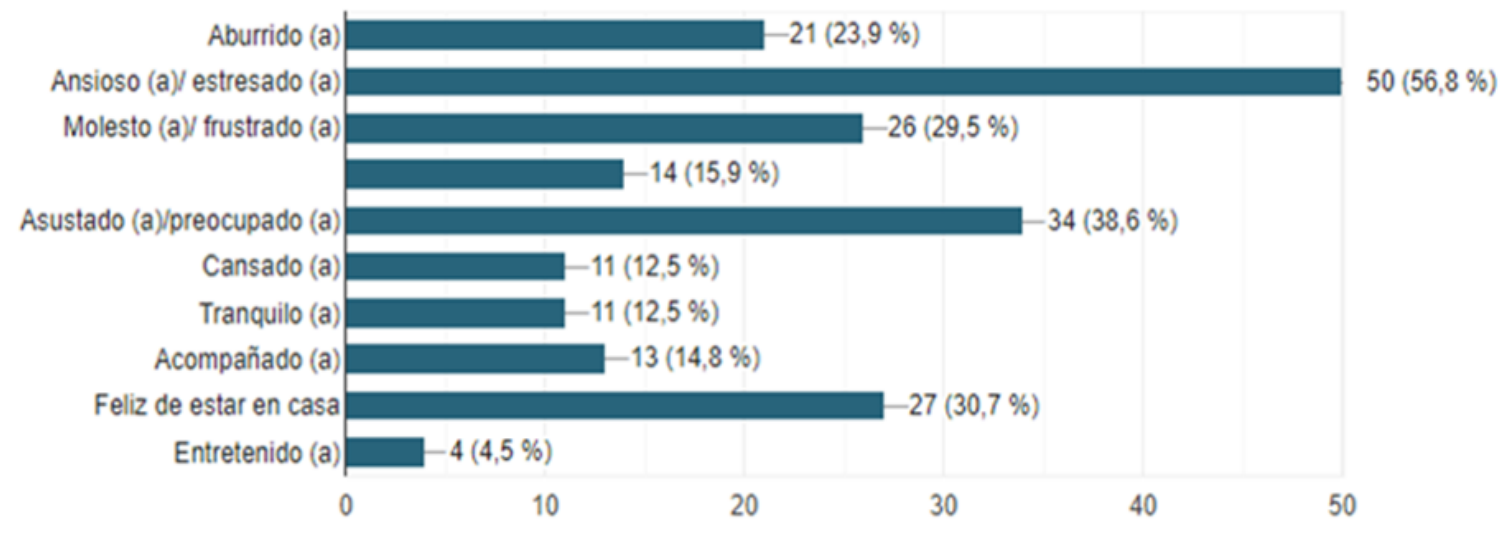

Fuente: Elaboración propia

Las principales dificultades que enfrentan los estudiantes en el escenario de la pandemia y los efectos sobre las estrategias de educación en línea se sintetizan en seis áreas: 1) carencia de conexión a internet y equipo de cómputo, 2) instrucciones del profesor 


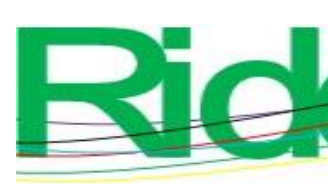

Revista Iberoamericana para la Investigación y el Desarrollo Educativo ISSN 2007 - 7467

poco claras e insuficientes, 3) ausencia de interactividad profesor-estudiante, 4) rigidez en los plazos y fechas de entrega, 5) exceso de tareas y actividades, 6) desempeño de actividades extraescolares prioritarias. De la misma manera, se describen las propuestas de acciones por parte de los profesores y los estudiantes para solucionar las dificultades que ambos actores enfrentan con el fin de mejorar las condiciones del proceso de enseñanzaaprendizaje hacia una educación en línea (tabla 1).

Tabla 1. Propuesta de acciones para reducir las dificultades que enfrentan los estudiantes y profesores para mejorar las condiciones del proceso de enseñanza-aprendizaje ante el escenario de la pandemia y la transición hacia una educación virtual

\begin{tabular}{|l|l|}
\hline Área de oportunidad & Propuesta de acción \\
\hline $\begin{array}{l}\text { Falta de equipo de cómputo y acceso a } \\
\text { internet. }\end{array}$ & $\begin{array}{l}\text { Los estudiantes deben establecer } \\
\text { estrategias que les permitan garantizar la } \\
\text { continuidad de su proceso de formación } \\
\text { profesional. Conocer dónde y cómo se } \\
\text { puede tener acceso a la red, solicitar apoyo } \\
\text { alos docentes, a sus compañeros de clase o } \\
\text { a la institución. }\end{array}$ \\
\hline $\begin{array}{l}\text { Instrucciones por parte de los docentes } \\
\text { insuficientes o poco claras que dificultan } \\
\text { las actividades del curso. }\end{array}$ & $\begin{array}{l}\text { Formación como docente en línea mediante } \\
\text { cursos de capacitación. }\end{array}$ \\
\hline $\begin{array}{l}\text { Falta de apoyo para el aprendizaje en línea } \\
\text { y ausencia de interactividad significativa } \\
\text { de los estudiantes con los profesores para } \\
\text { exponer sus dificultades. }\end{array}$ & $\begin{array}{l}\text { Asegurarse de que los materiales que se } \\
\text { proporcionan a los estudiantes como } \\
\text { recursos de aprendizaje son adecuados y } \\
\text { funcionales. Promover el dialogo y la } \\
\text { comunicación activa por diferentes canales } \\
\text { para captar las inquietudes de los } \\
\text { estudiantes. }\end{array}$ \\
\hline $\begin{array}{l}\text { Rigidez de los docentes en plazos y fechas } \\
\text { para que los estudiantes cumplan con las } \\
\text { actividades asignadas. }\end{array}$ & $\begin{array}{l}\text { Mostrar accesibilidad y flexibilidad con los } \\
\text { estudiantes. Demostrar empatía ante las } \\
\text { dificultades. }\end{array}$ \\
\hline $\begin{array}{l}\text { Asignación excesiva de actividades y } \\
\text { tareas durante el curso. }\end{array}$ & $\begin{array}{l}\text { Diseñar y programar de forma adecuada } \\
\text { los contenidos, actividades, tareas y } \\
\text { evaluaciones de las unidades de } \\
\text { aprendizaje. }\end{array}$ \\
\hline $\begin{array}{l}\text { actividades domésticas. } \\
\text { aestión por parte de los estudiantes para } \\
\text { disponer del espacio que cada unidad de } \\
\text { aprendizaje requiere, acordar con los } \\
\text { demás miembros de la familia el apoyo } \\
\text { para contar con el tiempo necesario para } \\
\text { atender todas las actividades académicas } \\
\text { sincrónicas y asíncronas. }\end{array}$ \\
\hline
\end{tabular}

Fuente: Elaboración propia 


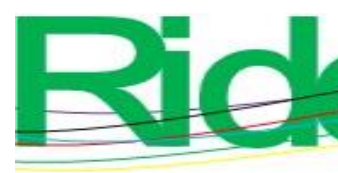

Revista Iberoamericana para la Investigación y el Desarrollo Educativo ISSN 2007- 7467

\section{Discusión}

Debido la presencia y la transmisión de la covid-19, las instituciones educativas incluidas las universidades públicas y privadas - suspendieron actividades académicas (Unesco, 2020a) para prevenir el contagio entre estudiantes y profesores, En el caso concreto del estado de Guerrero, se recurrió al confinamiento y al distanciamiento social para mitigar los efectos de la pandemia y reducir su impacto en la población, lo que obligó a cambiar la educación presencial por un modelo en línea para garantizar la conclusión del semestre febrero-julio de 2020 y el inicio de los semestres subsecuentes.

Estos eventos modificaron sustancialmente los esquemas de la educación tradicional y obligó a los estudiantes a enfrentar el reto de implementar un ambiente de aprendizaje, ubicado en el mismo espacio físico del hogar. Sin embargo, según los datos recabados en este trabajo, se puede afirmar que $75 \%$ de los encuestados no cuentan con las condiciones óptimas de los ambientes para el aprendizaje, ya que solo $25 \%$ de los estudiantes disponen de un espacio lo suficientemente agradable para lograr la concentración que garantice un aprendizaje efectivo. Adicionalmente, solo $52.3 \%$ cuenta con los materiales básicos para la realización de sus trabajos en línea (figura 1).

Otro elemento limitante en el proceso de enseñanza-aprendizaje es la relación del docente con los estudiantes. En tal sentido, no debe perderse de vista que la educación en los países desarrollados se masificó desde el siglo XIX, mientras que en México esto mismo ocurrió solo hasta la segunda mitad del siglo pasado. Además, la distancia entre el profesor y el estudiante se hace más notoria cuando las condiciones de pobreza de las comunidades marginadas en el estado de Guerrero impiden la comunicación efectiva entre ellos. Así lo reflejan los resultados de este estudio, los cuales muestran que solo uno de cada cuatro alumnos tiene contacto con el profesor $(26.1 \%)$ de cada asignatura y aproximadamente la mitad (56.8\%) tiene contacto con algún compañero de clase para resolver sus dudas (figura 1).

Este escenario no es diferente al de otros países en América Latina, como Ecuador, donde se observa el mismo patrón educativo en el que la disponibilidad de las TIC es una condición fundamental para garantizar la educación en línea y el desarrollo de las habilidades del estudiante (Corral y Corral, 2020). 


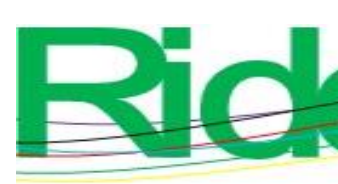

Revista Iberoamericana para la
Investigación y el Desarrollo Educativo
ISSN $2007-7467$

Estos resultados evidencian que las condiciones socioeconómicas que prevalecen en nuestro estado no favorecen los niveles de competitividad que se requieren para insertarnos en una sociedad en la que el conocimiento y la globalidad exigen una población mejor preparada.

En este contexto, el acompañamiento a los estudiantes como parte integral de la práctica docente, que tiene como objetivo asesorar y guiar al estudiante durante sus estudios, para contribuir con su formación integral, mediante la atención sistemática a un estudiante o a un grupo de ellos, también se encuentra en una situación poco favorecida, ya que los resultados de este estudio (figura 4) muestran que aproximadamente la mitad de los encuestados $(56.8 \%)$ recibieron acompañamiento algunas veces por parte de los profesores, mientras que tres de cada diez $(28.4 \%)$ recibieron acompañamiento casi siempre. Los resultados también indican que en términos reales uno de cada diez participantes $(11.3 \%)$ no recibió acompañamiento nunca $(1.1 \%)$ o casi nunca $(10.2 \%)$, que $85.1 \%$ recibió acompañamiento de manera ocasional, y solo $3.4 \%$ manifestó haber recibido acompañamiento siempre (figura 4).

La pandemia de la covid-19 trastocó la estructura organizacional de las instituciones educativas, pues migraron hacia un sistema de educación en línea (Secretaría de Educación Pública [SEP], 2020), sistema de enseñanza para el que no estaban preparados el personal docente ni los estudiantes. Esto representó un reto para ambos debido a que tuvieron que actualizarse en el uso de las tecnologías de la información, que comúnmente se utilizan en los modelos de enseñanza en línea (Shivangi, 2020). Ante este hecho, la universidad asumió el desafío y cambió súbitamente el enfoque pedagógico tradicional por un sistema de enseñanza-aprendizaje en línea (UAGro, 2020).

En este cambio repentino es importante reconocer las fortalezas, debilidades, oportunidades y desafíos del nuevo modelo de enseñanza implementado en este tiempo de crisis. Los resultados de la encuesta indican que, según la percepción de los estudiantes, el desempeño docente no fue el óptimo, ya que los resultados revelan que solo uno de cada dos profesores $(56.8 \%)$ se encuentra en la categoría de moderadamente preparados, mientras que uno de cada tres $(28.4 \%)$ están significativamente preparados, uno de cada $\operatorname{diez}(8 \%)$ se catalogaron como excelentemente preparados y $6.8 \%$ de los profesores están escasamente preparados (figura 5). 


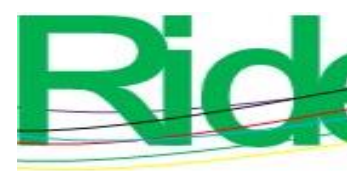

Revista Iberoamericana para la Investigación y el Desarrollo Educativo ISSN 2007 - 7467

Lo anterior sugiere que es necesario redoblar los esfuerzos para mejorar la capacitación de la planta docente con la finalidad de optimizar el desempeño académico y pasar a la siguiente categoría de significativamente preparados e incrementar la de excelentemente preparados, mediante la implementación de un programa de educación continua que abarque a la totalidad de los profesores que conforman la planta docente de la escuela.

De acuerdo con la percepción de los estudiantes, uno de cada dos profesores $(56.8 \%)$ está moderadamente preparado (figura 5). Este dato es consistente con la organización de los contenidos programáticos de las asignaturas, en donde uno de cada dos estudiantes $(50 \%)$ declara que los contenidos programáticos, tareas, dinámica y evaluaciones no fueron preparados con los criterios de planeación requeridos, y uno de cada tres $(31.8 \%)$ reconoce que solo algunos profesores mostraron un nivel de planeación acorde con el escenario de la educación en línea: planeación de los contenidos programáticos, tareas y evaluaciones (figura 6).

Los motivos que dificultaron el proceso de aprendizaje fueron los siguientes: los estudiantes percibieron que la cantidad de tareas y actividades solicitadas por los profesores fue excesiva (36.4\%), que el tiempo para entregar las tareas solicitadas no fue suficiente (43.2 \%), que los profesores no enviaron las explicaciones de forma clara para la realización de las actividades teóricas, prácticas y ejercicios $(45.5 \%$,) y que las tareas y actividades asignadas fueron muy difíciles (6.8\%) (figura 7).

En contraste con lo anterior, cuatro de cada diez estudiantes $(44.3 \%)$ percibieron que la cantidad de tareas y actividades asignadas fueron suficientes, tres de cada diez $(29.5 \%)$ que el tiempo para entregar las tareas a los profesores fue suficiente, y $4.5 \%$ consideró que las tareas y actividades fueron muy fáciles (figura 7).

Como puede observarse, los elementos que dificultan el proceso de enseñanzaaprendizaje son diversos. Actualmente, también se ha demostrado que muchos problemas psicológicos y consecuencias importantes en términos de salud mental (como estrés, ansiedad, depresión, frustración e incertidumbre) han surgido durante el brote de covid-19 (Duan y Zhu, 2020). La pandemia, en general, ha generado en los estudiantes sentimientos de aislamiento, frustración, aburrimiento, ansiedad y estrés (Pedró, 2020). Esto, sumado al cierre de las instituciones educativas, han tenido consecuencias que afectan la salud mental de los estudiantes (Unicef, 2020) y de la población en general (WHO, 2020). 


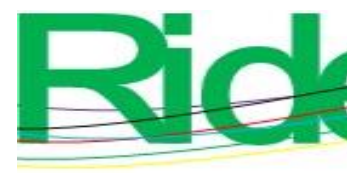

\section{Revista Iberoamericana para la Investigación y el Desarrollo Educativo ISSN $2007-7467$}

Se ha publicado información sobre el impacto psicológico de la pandemia y sus efectos sobre la población (pacientes afectados y no afectados, personal médico, niños y adultos mayores) (Cao et al., 2020; Duong et al., 2020), quienes han tenido que modificar de una u otra manera sus estilos de vida. Al respecto, los resultados del presente estudio muestran que la mitad de los encuestados $(53.4 \%)$ reconocen que han cambiado sustancialmente sus hábitos de vida a causa del confinamiento por la covid-19, y la otra mitad $(45.5 \%)$ los ha modificado en menor medida; es decir, prácticamente la totalidad de los encuestados $(98.9 \%$ ) refleja modificaciones en la conducta (figura 8). El hecho de quedarse en casa y llevar sus estudios en el mejor de los casos por medios electrónicos origina consecuencias emocionales asociadas con el sentimiento de aislamiento, frustración, aburrimiento, ansiedad o estrés (Duan y Zhu, 2020).

El tema de la salud mental que está emergiendo puede convertirse en el mediano plazo en un problema de salud pública. El impacto de la gravedad y las repercusiones medicas de la covid-19 en el mundo son de tal dimensión que podrían ocultar las consecuencias psicosociales derivadas del contagio y la transmisión de esta enfermedad. En la epidemia del síndrome respiratorio del medio oriente (MERS-CoV, por sus siglas en inglés), ocurrida en el 2012, se puso en evidencia que $40 \%$ de las personas afectadas requirieron de una intervención siquiátrica (Ferrel y Ryan, 2020). Se sabe que el aislamiento social y la movilidad restringida puede derivar en complicaciones psiquiátricas que varían desde síntomas aislados hasta el desarrollo de un trastorno mental, como insomnio, ansiedad, depresión o un trastorno de estrés postraumático (TEPT) (Chan et al., 2020).

En este contexto, se puede predecir, sin lugar a dudas, que la pandemia actual puede tener un efecto colateral potencial como la pérdida de la funcionalidad que puede compararse con un estado de duelo, y de persistir con un ánimo triste, se puede presentar un trastorno depresivo mayor (Chan et al., 2020). Los resultados de este estudio ponen en relieve dos aspectos importantes de la pandemia: la amenaza de la covid-19 como un problema de salud pública (figura 9) y los efectos del confinamiento sobre el estado emocional de los encuestados (figura 10). De esto se desprende que para hacer frente a la pandemia es necesario un enfoque que unifique en una causa común a la sociedad, pero se necesita la concientización y sensibilización para enfrentar esta situación de emergencia 


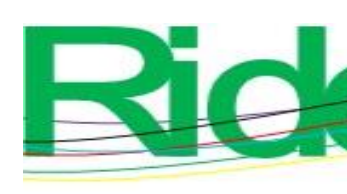

Revista Iberoamericana para la
Investigación y el Desarrollo Educativo
ISSN $2007-7467$

Los resultados obtenidos en el presente estudio muestran que en el escenario actual de la pandemia los problemas de la educación en línea siguen siendo muy similares (tabla 1) y se sintetizan en seis áreas principales: 1) carencia de conexión a internet y equipo de cómputo, 2) instrucciones del profesor poco claras e insuficientes, 3) ausencia de interactividad profesor-estudiante, 4) rigidez en los plazos y fechas de entrega, 5) exceso de tareas y actividades, y 6) desempeño de actividades prioritarias extraescolares (tabla 1).

En definitiva, las propuestas de acciones correctivas dependerán de la proactividad de los profesores y de los estudiantes para enfrentar las dificultades que afectan el desarrollo del proceso de enseñanza-aprendizaje ante el escenario de la pandemia y la transición hacia una educación en línea.

\section{Conclusiones}

A partir de los resultados obtenidos, se concluye que los recursos tecnológicos y el ambiente académico en el escenario de la educación en línea no son los óptimos para garantizar un aprendizaje efectivo debido a que los estudiantes no cuentan con espacios cómodos ni con los materiales básicos para el desarrollo de sus actividades académicas.

Es necesario reforzar la capacitación tanto de los estudiantes como de los profesores para garantizar la transición hacia la educación no presencial, ya que existen deficiencias por parte de los estudiantes y profesores con respecto a los conocimientos sobre plataformas virtuales y recursos de la educación en línea.

Sobre la interconectividad, más de la mitad refiere que no cuenta con equipo de cómputo ni acceso a internet y/o carecen de capacitación sobre competencias digitales.

En relación con la organización de los contenidos programáticos de las asignaturas, tareas, dinámicas y evaluaciones, los encuestados refieren que no fueron preparados con los criterios de planificación y conocimiento en entornos virtuales de aprendizaje.

En cuanto a los efectos de la pandemia, no solo se relacionaron con la salud física, sino que también reportaron modificaciones en la conducta, repercusiones en la salud mental, en el estado emocional y en el rendimiento académico; lo anterior relacionado con la percepción de considerar la situación como un evento grave o extremadamente grave que originó modificaciones en los hábitos de vida, síntomas de ansiedad, estrés, angustia y preocupación.

Las principales dificultades que los estudiantes enfrentan y que limitan la educación 


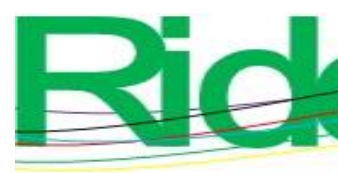

Revista Iberoamericana para la
Investigación y el Desarrollo Educativo
ISSN $2007-7467$

en línea, pero que además ponen en riesgo la continuidad educativa, son los siguientes: a) el acceso limitado a las plataformas educativas digitales, b) la brecha de equidad tecnológica entre las diferentes localidades geográficas del estado de Guerrero, c) los escasos conocimientos que poseen los estudiantes y docentes sobre las diferentes herramientas digitales disponibles para la educación en línea, d) las pocas iniciativas fuera de línea para responder y resolver la problemática relacionada con los estudiantes universitarios sin acceso a internet y e) los efectos psicosociales del confinamiento asociados a la covid-19.

\section{Futuras líneas de investigación}

La propuesta federal de la Anuies plantea generar nuevas líneas de acción que estructuren y definan los nuevos modelos educativos, la emergencia de nuevos paradigmas y mejores prácticas educativas. Ante esta perspectiva, se han implementado diversas estrategias para garantizar la continuidad educativa en la transición de la modalidad de las clases presenciales a la modalidad en línea, e identificar áreas que permitan optimizar el aprendizaje y la innovación de la educación en el nuevo modelo educativo. Los resultados y las herramientas metodológicas utilizadas en este estudio pueden servir como una plataforma para la realización de diagnósticos situacionales de la educación en línea que permitan detectar las fortalezas y fragilidades en el proceso de enseñanza-aprendizaje y coadyuven a incrementar la calidad educativa.

\section{Agradecimientos}

Agradecemos la participación y el apoyo técnico de Fernando Rosario Domínguez en la sistematización de los resultados y el procesamiento de las imágenes. 


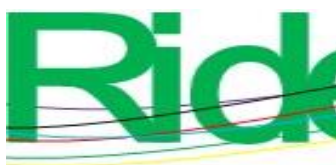

Revista Iberoamericana para la
Investigación y el Desarrollo Educativo
ISSN $2007-7467$

\section{Referencias}

Asociación Nacional de Universidades e Instituciones de Educación Superior [Anuies]. (2020). Sugerencias para mantener los servicios educativos curriculares durante la etapa de emergencia sanitaria provocada por el COVID-19. Nayarit, México: Universidad Autónoma de Nayarit. Recuperado de https://piida.uan.mx/blog/sugerencias-para-mantener-los-servicios-educativosdurante-la-emergencia-sanitaria

Ballén Cifuentes, O. A., Baquero Garzón, L. A., Padilla Berrío, M. J. y Bernal Sáchica, D. F. (2021). Balance de la pandemia: lecciones aprendidas de la coyuntura frente a las medidas de emergencia sobre el sector educativo en Bogotá. Revista Educación y Ciudad, (41), e2575. Doi: https://doi.org/10.36737/01230425.n41.2575

Borges, F. (2005). La frustración del estudiante en línea. Causas y acciones preventiva. Digithum, (7). Recuperado de http://www.uoc.edu/digithum/7/dt/esp/borges.pdf

Cao, W., Fang, Z., Hou, G., Han, M., Xu, X. and Dong, J. (2020). The psychological impact of the COVID-19 epidemic on college students in China. Psychiatry Res, 278, 1- 12. Doi https://doi.org/10.1016/j.psychres.2020.112934

Chan, J. F, Yuan, S., Kok, K. H., To, K. K., Chu, H., Yang, J. et al. (2020). A familial cluster of pneumonia associated with the 2019 novel coronavirus indicating personto-person transmission: a study of a family cluster. The Lancet, 395(10223), 514-23. Retrieved from http://www.ncbi.nlm.nih.gov/pmc/articles/pmc7159286/

Comisión Nacional para la Mejora Continua de la Educación [Conamece] (2020). 10 sugerencias para la educación durante la emergencia por COVID-19. Ciudad de México, México: Gobierno de México. Recuperado de https://www.gob.mx/mejoredu/articulos/10-sugerencias-para-la-educacion-durantela-emergencia-por-covid-19

Corral, Y. and Corral, I. (2020). Una mirada a la educación a distancia y uso de las TICs en tiempos de pandemia. Revista Eduweb, 14(1), 143-150. Recuperado de https://revistaeduweb.org/index.php/eduweb/article/view/14

Cucinotta, D. and Vanelli, M. (2020). WHO Declares COVID-19 a Pandemic. Acta Biomedica, $\quad 91(1)$. 157-160. $\quad$ Retrieved from https://www.mattioli1885journals.com/index.php/actabiomedica/article/view/9397/8 659 


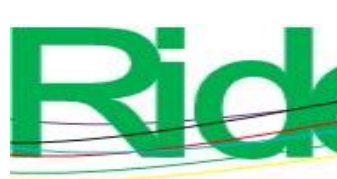

Revista Iberoamericana para la
Investigación y el Desarrollo Educativo
ISSN $2007-7467$

Delgado, P. (6 de abril de 2020). Padres, alumnos y docentes enfrentan los retos de adaptarse a la educación en línea. Observatorio de Innovación Educativa. Recuperado de https://observatorio.tec.mx/edu-news/educacion-online-retosescuela-en-casa

Dryhurst, S., Schneider, C. R., Kerr, J., Freeman, A. L. J., Recchia, G., van der Bles, A. M. and van der Linden, S. (2020). Risk perceptions of COVID-19 around the world. Journal of Risk Research,23(7-8). 994-1006. Doi; https://doi.org/10.1080/13669877.2020.1758193

Duan, L and Zhu, G. (2020). Psychological interventions for people affected by the COVID-19 epidemic. Lancet Psychiatry, 7(4), 300-302. Doi; https://doi.org/10.1016/S2215-0366(20)30073-0

Duong, V., Luo, J., Pham, P., Yang, T. and Wang, Y. (2020). The Ivory Tower Lost: How College Students Respond Differently than the General Public to the COVID-19 Pandemic. International Conference on Advances in Social Networks Analysis and Mining IEEE/ACM (ASONAM). 126-130. Retrieved from https://ieeexplore.ieee.org/abstract/document/9381379

Ferrel, M. and Ryan, J. (2020). The Impact of COVID-19 on Medical Education. Cureus, 12(3), e7492. Retrieved from https://www.ncbi.nlm.nih.gov/pmc/articles/PMC7193226/

Giannini, S. (2020). COVID-19 y educación superior: de los efectos inmediatos al día después. Revista Latinoamericana de Educación Comparada, 11(17). 1-57. Recuperado de https://dialnet.unirioja.es/ejemplar/552807

Han, C. (2008). Doing Qualitative Research Using Your Computer: A Practical Guide. Computer Center. Retrieved from https://www.semanticscholar.org/paper/DoingQualitative-Research-Using-Your-Computer\%3A-A Hahn/066ae166f754445fd677f4499aafe31ea03c609e

Khan, S., Siddique, R., Li, H., Ali, A., Shereen, M. A., Bashir, N. and Xue, M. (2020). Impact of coronavirus outbreak on psychological health. Journal of Lobal Health, 10(1). Doi: https://doi.org/10.7189/jogh.10.010331

Marín, M. A. (8 de junio de 2020). Educación virtual: lecciones aprendidas por los maestros en tiempos de pandemia. Estamos en línea, noticias de tecnología e innovación. Recuperado de https://news.microsoft.com/es-xl/educacion-virtual- 


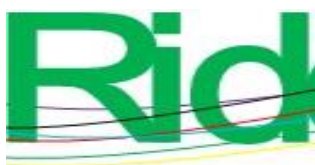

Revista Iberoamericana para la
Investigación y el Desarrollo Educativo
ISSN $2007-7467$

United Nations Educational, Scientific and Cultural Organization [Unesco] (2020c). El derecho a la educación. Recuperado de https://es.unesco.org/themes/derecho-aeducacion

Universidad Autónoma de Guerrero [UAGro] (16 de marzo de 2020)._Clases virtuales: la estrategia de la UAGro para salvar el semestre. Recuperado de https://uagrocontigo.com/2020/04/16/clases-virtuales-la-estrategia-de-la-uagro-parasalvar-el-semestre/

Valverde-Berrocoso, J., Fernández-Sánchez, M. R., Revuelta Domínguez, F. I. and SosaDíaz, M. J. (2021). The educational integration of digital technologies preCovid-19: Lessons for teacher education. PloS one, 16(8), 1-22. Doi: https://doi.org/10.1371/journal.pone.0256283

Van-Dijk, T. (2000). El discurso como estructura y proceso: estudios sobre el discurso. Gedisa. Recuperado de: https://www.academia.edu/7929009/Rese\%C3\%B1a_de_T_van_Dijk_El_discurso_ como_estructura_y_proceso_Estudios_del_discurso_introducci\%C3\%B3n_multidis ciplinaria_2001_

Villafuerte, P. (19 de marzo de 2020). Observatorio de innovación educativa. Tecnológico de Monterrey. Educación en tiempos de pandemia: COVID-19 y equidad en el aprendizaje. Recuperado de https://observatorio.tec.mx/edu-news/educacion-entiempos-de-pandemia-covid19

World Health Organization [WHO]. (2020). Mental health and psychosocial considerations during the COVID-19 outbreak. Retrieved from https://www.who.int/docs/defaultsource/coronaviruse/mental-health-considerations.pdf?sfvrsn=6d3578af_2 


\begin{tabular}{|l|l|}
\hline Rol de Contribución & Autor (es) \\
\hline Conceptualización & Delia Inés Dominguez García, Rodrigo Rosario Cruz (igual) \\
\hline Metodología & Delia Inés Domínguez-García, Rodrigo Rosario Cruz (igual) \\
\hline Software & NA \\
\hline Validación & $\begin{array}{l}\text { Delia Inés Domínguez-García, Fernando Romero Torres, } \\
\text { Rodrigo Rosario Cruz (igual) }\end{array}$ \\
\hline Análisis Formal & $\begin{array}{l}\text { Delia Inés Domínguez-García, Fernando Romero Torres, } \\
\text { Rodrigo Rosario Cruz (igual) }\end{array}$ \\
\hline Investigación & $\begin{array}{l}\text { Delia Inés Domínguez-García (principal), Rodrigo Rosario } \\
\text { Cruz (apoya) }\end{array}$ \\
\hline Recursos & $\begin{array}{l}\text { Delia Inés Domínguez-García, Fernando Romero Torres, } \\
\text { Rodrigo Rosario Cruz (igual) }\end{array}$ \\
\hline $\begin{array}{l}\text { Curación de datos } \\
\text { Escritura - Preparación del } \\
\text { borrador original }\end{array}$ & Delia Inés Domínguez-García, Rodrigo Rosario Cruz (igual) \\
\hline Escritura - Revisión y & $\begin{array}{l}\text { Delia Inés Domínguez-García, Fernando Romero Torres, } \\
\text { Rodrigo Rosario Cruz (igual) }\end{array}$ \\
\hline Supervisión & Delia Inés Domínguez-García \\
\hline Administración de Proyectos & Rodrigo Rosario Cruz, Rodrigo Rosario Cruz (igual) \\
\hline Adquisición de fondos & Delia Ines Dominguez García \\
\hline
\end{tabular}

